\title{
Numerical study on a polymer-shelled microbubble submerged in soft tissue
}

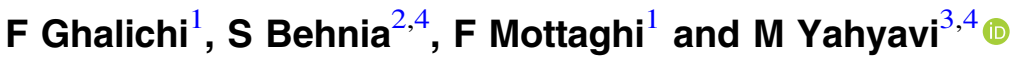 \\ ${ }^{1}$ Department of Biomedical Engineering Division of Biomechanics, Sahand University of Technology, \\ Sahand New Town, Tabriz, Iran \\ ${ }^{2}$ Department of Physics, Urmia University of Technology, Urmia, Iran \\ ${ }^{3}$ Department of Physics, Bilkent University 06800, Ankara, Turkey \\ E-mail: s.behnia@sci.uut.ac.ir and yahyavi.mohamad@gmail.com
}

Received 21 April 2020, revised 20 June 2020

Accepted for publication 29 June 2020

Published 7 July 2020

\begin{abstract}
Ultrasound contrast agents have been recently utilized in therapeutical implementations for targeted delivery of pharmaceutical substances. Radial pulsations of the encapsulated microbubbles under the action of an ultrasound field are complex and high nonlinear, particularly for drug and gene delivery applications with high acoustic pressure amplitudes. The dynamics of a polymer-shelled agent are studied through applying the method of chaos physics whereas the effects of the outer medium compressibility and the shell were considered. The stability of the ultrasound contrast agent is examined by plotting the bifurcation diagrams, Lyapunov exponent, and time series over a wide range of variations of influential parameters. The findings of the study indicate that by tuning the shear modulus of surrounding medium and shell viscosity, the radial oscillations of microbubble cluster undergoes a chaotic unstable region as the amplitude and frequency of ultrasonic pulse are increased mainly due to the period doubling phenomenon. Furthermore, influences of various parameters which present a comprehensive view of the radial oscillations of the microbubble are quantitatively discussed with clear descriptions of the stable and unstable regions of the microbubble oscillations for typical therapeutic ultrasound pulses.
\end{abstract}

Keywords: bubble dynamics, chaos theory, nonlinear dynamics

(Some figures may appear in colour only in the online journal)

\section{Introduction}

Ultrasound contrast agents (UCAs) are coated microbubbles by a stabilizing layer such as albumin, polymer or lipids which are usually formed with a high-molecular-weight gas [1-3]. These agents are originally designed for diagnostic ultrasound imaging (for liver imaging, cardiac and other organs) since they are highly detectable with ultrasound imaging due to their great scattering properties, hence this acoustic trait caused to the progression of more sensitive imaging methods $[4,5]$. Recently, in addition to diagnostic implementations, their employment in the biomedical field is translating to therapeutic applications [6] such as drug and gene delivery [7, 8], sonothrombolysis [9], opening of the blood-brain barrier and drug delivery to the CNS [10-12].

\footnotetext{
${ }^{4}$ Authors to whom any correspondence should be addressed.
}

Indeed, they are employed as transporters of pharmaceutical agents to carry them into the site of interest to deliver their cargo just where it is needed by applying a focused ultrasound field [8, 13-15]. This novel method has emerged immense clinical potentials such as minimizing drug-related toxicity to the healthy cells and tissues, drug dosage modifications, promoting transmembrane and extravascular drug transport, preventing drug-drug interactions, decreasing costs for the patient, additionally, transference and release can be visualized with real-time ultrasound and as a whole result treatment efficacy will be enhanced [16-18].

UCAs undergo complex dynamic behaviors while they are exposed to an ultrasound field [19]. Depending on the applied acoustic amplitudes, the microbubble structure and the properties of the host media, they will respond linear or nonlinear pulsations [20-22]. Several investigations also demonstrated that a microbubble and microbubble cluster 
behavior in ultrasonic fields [23-25]. Fundamental perception of UCAs dynamics and precisely predicting their behavior will promote their diagnostic and therapeutic capabilities; indeed a quantitative understanding of UCAs dynamics is a necessary step to attain a better hardware design and successful clinical applications. Many sophisticated theoretical treatments for describing the coated microbubble response in an ultrasound field have been performed whereas most of the presented models are on the foundation of the RayleighPlesset (RP) equation form. De Jong and co-workers [26, 27] introduced the first theoretical model that considers the encapsulation as a viscoelastic solid shell, as well as a damping coefficient term, is added to the RP equation. Church [28] presented a more accurate model by considering the shell thickness to describe the effects of the shell on UCA behavior. Morgan (see also Zheng) [29, 30] and Allen [31] offered their models for thin and thick encapsulated microbubbles, respectively. Another rigorous model which treats the outer medium as a slightly compressible viscoelastic liquid is due to Khismatullin and Nadim [32]. Chatterjee and Sarkar [33] attempted to take account of the interfacial tension at the microbubble interface with infinite small shell thickness. Sarkar [34] improved this model to contain the surface elasticity by using a viscoelastic model. Stride and Saffari [35] demonstrated the presence of blood cells and the adhesion of them to the shell have a negligible effect. Tamadapu and coworkers [36, 37] investigated an air-filled thick polymer encapsulated nonspherical microbubble suspended in bulk volume of water. Marmottant [38] exhibited a simple model for the dynamics of phospholipid-shelled microbubbles while taking account of a buckling surface radius, shell compressibility, and a break-up shell tension. Doinikov and Dayton [39] refined the church model and also considered the translational motion of the UCA. Shengping Qin and Katherine W. Ferrara [40] have presented a model to explain the radial oscillations of UCAs by considering the effects of liquid compressibility, the surrounding tissue, and the shell. Although the aforementioned discussion expressed that considerable efforts have been performed, nonlinear dynamics of encapsulated microbubble by considering variations in different effective parameters is not fully realized by any means $[41,42]$ and require supplemental developments. The nonlinear nature of the equation needs specialized tools for analyzing because linear and analytical solutions are inadequate. Lauterborn and his colleagues [43-45] have made great contributions by introducing the method of chaos physics to a model of a driven spherical gas bubble in water to determine its dynamic properties, especially its resonance behavior and bifurcation structure. Also, based on the previous works, the chaotic behavior of free bubbles observed both theoretically and experimentally [46-50], but this is not investigated for the case of UCAs, and it will be helpful to survey from this point of view because the method of chaos physics provides extensive knowledge about rich nonlinear dynamical systems. Moreover, neglecting liquid compressibility is not suitable for high-pressure amplitudes where the wall velocity of the agent is equal to the speed of sound in liquid [31], so the effects of liquid compressibility on the microbubble dynamics should be considered [40, 41].

In this paper, the effects of substantial parameters that influence the UCA dynamics are studied in a large domain applying method of chaos physics [43-45] and considering the compressibility of the outer medium and the shell. It will represent comprehensive information about extremely nonlinear pulsations of UCAs, particularly for drug and gene delivery applications.

\section{Mathematical model: dynamics of a coated spherical microbubble}

The theoretical description of radial motion for a spherical encapsulated microbubble submerged in blood or tissue has been derived by Qin and Ferrara [40] which is utilized for numerical simulation. This justified equation also explains the effects of variations of the shell and the surrounding tissue on the UCA behavior and is given by:

$$
\begin{aligned}
& {\left[\rho_{L}\left(1-\frac{\dot{R}_{2}}{c}\right)+\rho_{S}\left(1+\frac{\dot{R}_{2}}{c}\right)\left(\frac{R_{2}}{R_{1}}-1\right)\right] R_{2} \ddot{R}_{2}} \\
& +\left\{\frac{3}{2} \rho_{L}\left(1-\frac{1}{3} \frac{\dot{R}_{2}}{c}\right)\right. \\
& \left.+\rho_{S}\left(1+\frac{\dot{R}_{2}}{c}\right)\left[-\frac{3}{2}+2\left(\frac{R_{2}}{R_{1}}\right)-\frac{1}{2}\left(\frac{R_{2}}{R_{1}}\right)^{4}\right]\right\} \dot{R}_{2}^{2} \\
& =\left(1+\frac{\dot{R}_{2}}{c}\right)\left\{p_{g}(t)-\frac{2 \sigma_{1}}{R_{1}}\right. \\
& -\frac{2 \sigma_{2}}{R_{2}}-\frac{4}{3} G_{S}\left[1-\left(\frac{R_{20}}{R_{2}}\right)^{3}\right] \frac{R_{S}}{R_{2}^{3}-R_{S}} \\
& -4 \mu_{S} \frac{R_{S}}{R_{2}^{3}-R_{S}} \frac{\dot{R}_{2}}{R_{2}}-\frac{4}{3} G_{L}\left[1-\left(\frac{R_{20}}{R_{2}}\right)^{3}\right] \\
& \left.-4 \mu_{L} \frac{\dot{R}_{2}}{R_{2}}-p_{0}-p_{i}(t)\right\}-3 \gamma \frac{\dot{R}_{2}}{c}\left(\frac{R_{2}}{R_{10}}\right)^{3} \frac{p_{g}(t)}{\left(\frac{R_{1}}{R_{10}}\right)^{3}-\frac{b}{V_{m}}} \text {. }
\end{aligned}
$$

where $p_{i}(t)=P_{a} \sin (2 \pi f t)$ is the ultrasound pressure at infinity. Also, the pressure-volume relation, $p_{g}$, is defined as follows

$$
p_{g}(t)=\left(p_{0}+\frac{2 \sigma_{1}}{R_{10}}+\frac{2 \sigma_{2}}{R_{20}}\right)\left[\frac{1}{\left(\frac{R_{1}}{R_{10}}\right)^{3}-\frac{b}{V_{m}}}\right]^{\gamma}
$$

The equation (1) is applied to describe nonlinear oscillations of a polymer-shelled agent versus variations of several important parameters. This model was developed to describe the dynamics of UCAs in vivo while taking account of the effects of the surrounding tissue, the shell tissue, and liquid compressibility. In the literature, the correction term for compressibility has different forms for different considerations. In this work, we choose the form $(R / c)\left(d p_{g}(t) / d t\right)$ as in [51]. Since the time derivative of the driving pressure 
Table 1. Physical constants parameters for polymer-shelled agent [22, 35].

\begin{tabular}{lccc}
\hline Symbol & Parameter & Value & Unit \\
\hline$\rho_{S}$ & Shell density & 1150 & $\frac{\mathrm{kg}}{\mathrm{m}^{3}}$ \\
$\rho_{L}$ & Liquid density & 1060 & $\frac{\mathrm{kg}}{\mathrm{m}^{3}}$ \\
$\mu_{L}$ & Liquid viscosity & 0.015 & $\mathrm{~Pa} \mathrm{~s}$ \\
$\sigma_{1}$ & Surface tension at inner radius & 0.04 & $\mathrm{~N} \mathrm{~m} \mathrm{~m}^{-1}$ \\
$\sigma_{2}$ & Surface tension at outer radius & 0.056 & $\mathrm{~N} \mathrm{~m} \mathrm{~m}^{-1}$ \\
$R_{10}$ & Equilibrium inner radius of agent & 2.3750 & $\mu \mathrm{m} \mathrm{[40]}$ \\
$R_{20}$ & Equilibrium outer radius of agent & 2.5 & $\mu \mathrm{m} \mathrm{[40]}$ \\
$p_{0}$ & Hydrostatic pressure & 1.01 & $\times 10^{5} \mathrm{~Pa}$ \\
$c$ & Sound speed in liquid & 1540 & $\frac{\mathrm{m}}{\mathrm{s}}$ \\
$b$ & Van der Waals constant & 0.1727 & $\frac{\mathrm{l}}{\mathrm{mol}}$ \\
$V_{m}$ & Universal molar volume & 22.4 & $\frac{\mathrm{l}}{\mathrm{mol}}$ \\
$\gamma$ & Polytropic gas exponent & 1.4 & \\
\hline
\end{tabular}

Table 2. Physical varied parameters for polymer-shelled agent [22, 35].

\begin{tabular}{lcc}
\hline Parameter & Range of value & Unit \\
\hline Driving pressure & $0<P_{a}<2$ & $\mathrm{MPa}$ \\
Driving frequency & $0.5<f<5$ & $\mathrm{MHz}$ \\
Shear modulus of surrounding medium & $0<G_{L}<1.5$ & $\mathrm{MPa}$ \\
Shell viscosity & $0<\mu_{S}<5$ & $\mathrm{~Pa} \mathrm{~s}$ \\
Shear modulus of shell & $0<G_{S}<200$ & $\mathrm{MPa}$ \\
Shell thickness & $0<R_{S}<0.15$ & $\mu \mathrm{m}$ \\
\hline
\end{tabular}

$\left(d p_{g}(t) / d t\right)$ is small and not dominant for violent oscillation. The assumption used in this paper is that the shell thickness is finite and the shell material behaves as a Voigt viscoelastic solid. The Qin-Ferrara is similar to the Church model [28], but, the Church shell elastic term is valid only for small deformation since in the Qin-Ferrara's model, the shell elastic term is stated to be valid for finite deformation of the shell.

\subsection{Variables and its domain}

The evolution of microbubble dynamics corresponds to the different parameter, which should be explained separately. As the inner and outer radius of the agent is described with $R_{1}$ and $R_{2}$, then $\dot{R}_{1}$ and $\dot{R}_{2}$ are the inner and outer wall velocity of the agent, respectively. $\ddot{R}_{2}$ is the outer wall acceleration of the agent. Naturally, $R_{10}$ and $R_{20}$ used as initial outer and inner radius of bubbles. Also, $R_{S}=R_{20}^{3}-R_{10}^{3}$ and $\rho_{L}$ is the density of the liquid and $\rho_{S}$ is the Shell density. $c$ is the speed of sound in the liquid. $\sigma_{1}$ is the inner surface tension, $\sigma_{2}$ is the outer surface tension, $p_{g}$ is the gas pressure within the agent. $G_{S}$ is the shear modulus, $G_{L}$ is the shear modulus of the surrounding medium which represent the stiffness of the surrounding tissue. $\mu_{L}$ is the viscosity of the liquid, $\mu_{s}$ is shell viscosity. Finally, $f$ is the ultrasound center frequency, $b$ is the van der Waals constant, $V_{m}$ is the universal molar volume, $p_{0}$ is the hydrostatic pressure. The introduced constants and varied parameter values for polymer-shelled agent are summarized in tables 1 and 2 [22, 35]. Actually, in this paper, all physical parameters were kept constant at values given in table 1 . This model assumes a single microbubble dispersed within an infinite medium, with no boundary conditions, no bubble-bubble interactions, no flow. Furthermore, bubblebubble interactions, primary and secondary Bjerknes forces, clustering, coalescence, and generally ultrasound-propelled motions are ignored. Most importantly, bubble destruction, gas diffusion in every acoustic cycle, polymer shedding, and other shell-modifying phenomena are not taken into account here.

\section{Results}

For a better visualization of the evolution of the effect of acoustic pressure alterations on microbubble dynamics, radial motion of a UCA is investigated versus a prominent domain of acoustic pressure from $10 \mathrm{kPa}$ to $2 \mathrm{MPa}$. Figuress 1(a)-(f) show the bifurcation diagrams and Lyapunov exponent $(\lambda)$ $[48,52,53]$ of the normalized microbubble radius against acoustic pressure as the control parameter for several values of applied frequency of the ultrasound field which they are $0.6,1,1.5,1.8,2.2$ and $2.8 \mathrm{MHz}$, respectively. In each one stable and chaotic pulsations can be observed, regarding the sign of the corresponding Lyapunov exponent. The existence of the negative or positive Lyapunov exponent indicates the non-chaotic or chaotic behavior. It is perceived that by raising pressure the microbubble stability is reduced and chaotic oscillations will be evident which this trend can be confirmed by other works $[38,53,54]$. Regarding figures $1(\mathrm{a})-(\mathrm{f})$, the microbubble experiences distinctive behaviors in different frequency while the control parameter (acoustic pressure) is increasing. As it is followed in figures 1(a)-(f), by increasing the center frequency the microbubble stability is enlarged in superior driving pressures which is in a good agreement with the work of [55]. This is obvious in figure 1(f), where the accessibility to the stable range concerning variations of pressure has the maximum extent.

According to figure $1(\mathrm{~d})$, the radial motion of the microbubble in frequency $1.8 \mathrm{MHz}$ manifests stable behavior of period one till $352 \mathrm{kPa}$ which is followed by a period doubling up to $615 \mathrm{kPa}$, after that the system demonstrates a period four for a small interval and it is pursued by the first chaotic window in $680 \mathrm{kPa}(\lambda>0)$. Then the microbubble exhibits its periodic behavior again before the next jump to chaos in $867 \mathrm{kPa}$. These intermittent transitions between chaotic oscillations and stable behavior persist until 1.3 MPa, afterward, the system turns into severely chaotic oscillations which continues to the termination of pressure interval, i.e., $2 \mathrm{MPa}$. This behavior is also observed experimentally in [56]. The microbubble experience more stability in a broad domain of driving pressure and chaotic pulsations and the expansion ratio of the UCA is reduced while the applied frequency is higher (see figure 1(f)).

Also for studying the effect of frequency alterations on microbubble dynamics, the dynamical behavior of UCA is inspected by considering the ultrasound frequency as the control parameter which varies from $600 \mathrm{kHz}$ to $5 \mathrm{MHz}$, the 

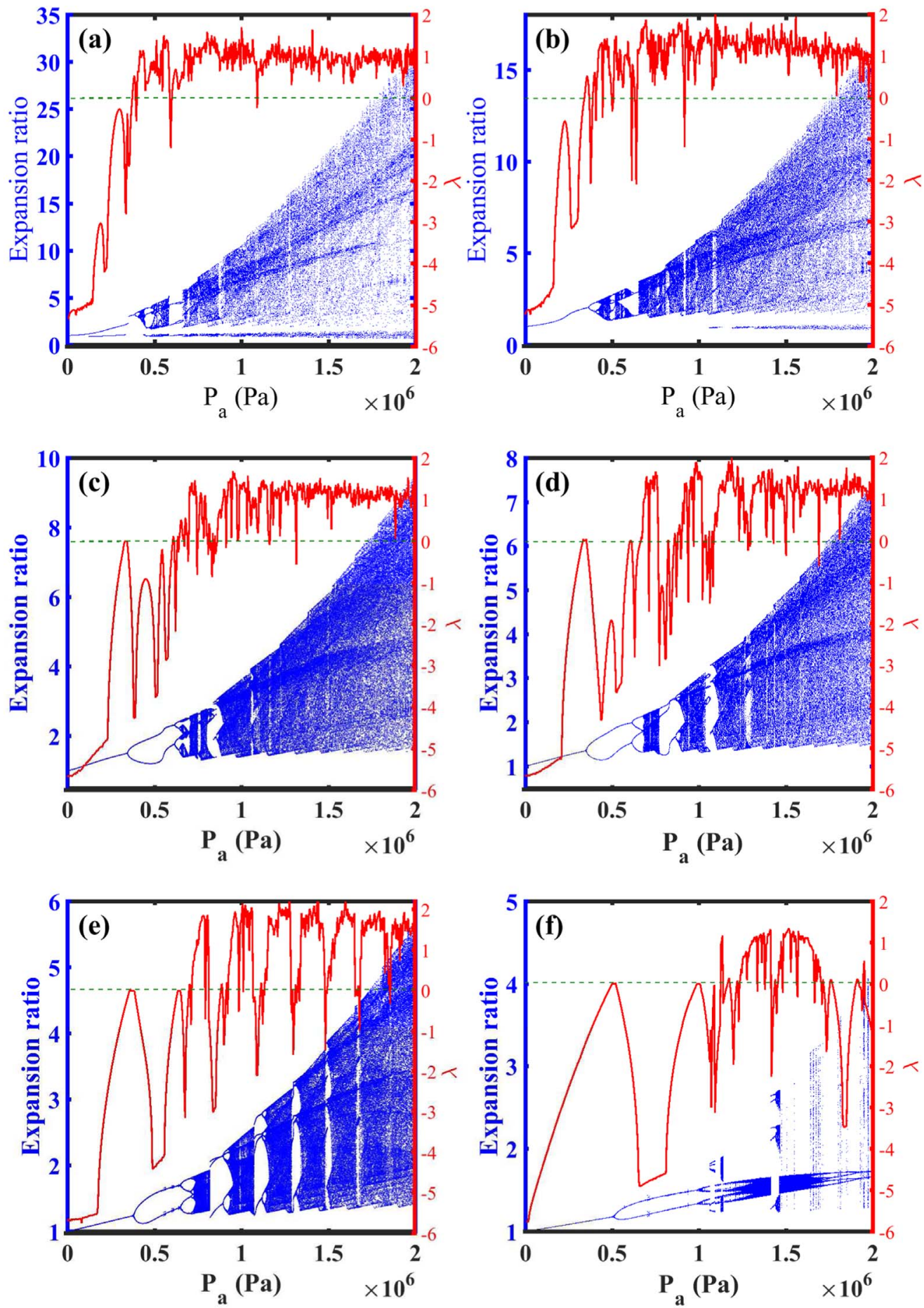

Figure 1. Bifurcation diagrams (Expansion ratio-blue dot points) and Lyapunov exponent ( $\lambda$-red solid line) of normalized microbubble radius versus driving pressure within blood $\left(G_{L}=0 \mathrm{MPa}\right.$ ) with $\mu_{s}=0.45 \mathrm{~Pa} \mathrm{~s}$, and $G_{S}=11.7 \mathrm{MPa}$ while the frequency is (a) $0.6 \mathrm{MHz}$, (b) $1 \mathrm{MHz}$, (c) $1.5 \mathrm{MHz}$, (d) $1.8 \mathrm{MHz}$, (e) $2.2 \mathrm{MHz}$ and (f) $2.8 \mathrm{MHz}$. All other physical parameters were kept constant at values given in table 1 .

corresponding bifurcation diagrams and Lyapunov exponent of the normalized microbubble radius is shown in figure 2(a)(f) for the applied pressure values of $0.3,0.5,0.9,1.2,1.7$, 2.2 $\mathrm{MPa}$, respectively.

The stable behavior of microbubble is presented for the low amplitude of pressure $(\lambda<0)$, i.e., $0.3 \mathrm{MPa}$ (figure 2(a)). The chaotic behavior $(\lambda>0)$ of UCA appears by increasing the values of applied pressure (figure 2(b)), and the microbubble shows more chaotic oscillations as the pressure is intensifying (figures $2(\mathrm{~b})-(\mathrm{f})$ ) which this phenomenon is seen in $[21,57]$. It is seen in all figures 2(a)-(f) that, the magnitude of pulsations reduces significantly and the chaotic region becomes smaller when the control parameter (frequency) is increasing, and the UCA shows the stable behavior of period one which reveals the stabilizing property of superior frequencies which is confirmed in [31]. It is seen in 

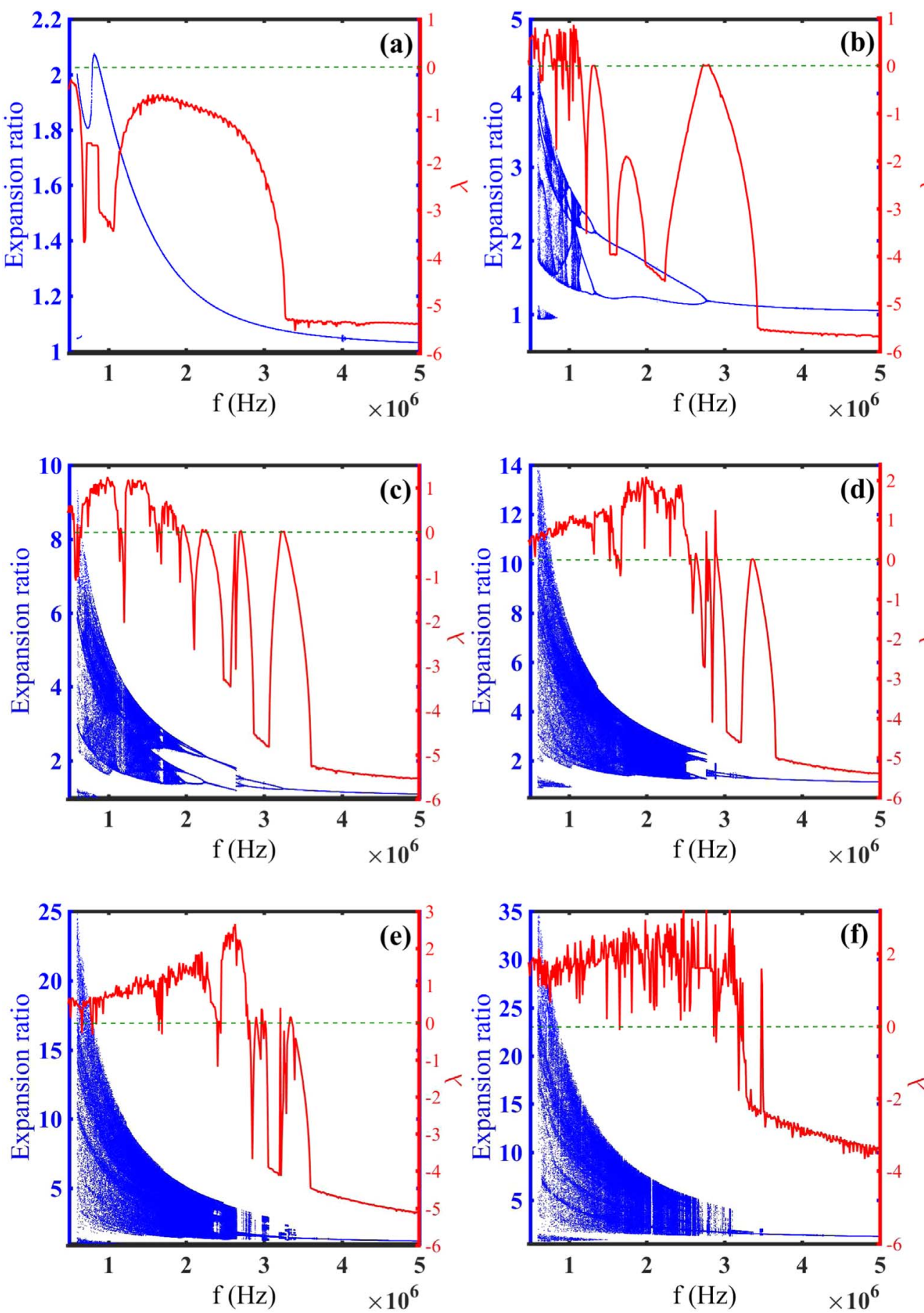

Figure 2. Bifurcation diagrams (Expansion ratio-blue dot points) and Lyapunov exponent ( $\lambda$-red solid line) of normalized microbubble radius versus driving frequency whit $G_{L}=0 \mathrm{MPa}, \mu_{s}=0.45 \mathrm{~Pa} \mathrm{~s}$, and $G_{S}=11.7 \mathrm{MPa}$ while the acoustic pressure is (a) $0.3 \mathrm{MPa}$, (b) $0.5 \mathrm{MPa}$, (c) $0.9 \mathrm{MPa}$, (d) $1.2 \mathrm{kPa}$, (e) $1.7 \mathrm{MPa}$ and (f) $2.2 \mathrm{MPa}$.

all of them (figures 2(a)-(f)) that UCA goes to stable manner at high values of frequency and microbubbles exposed to superior pressures become stable at superior frequencies.

The mechanical characteristics of the medium that surrounds the UCA are varied with kind of tissue and its composition. Therefore, the effects of shear modulus of tissue on microbubble behavior are studied by considering shear modulus variations from 0 to $1.5 \mathrm{MPa}$. Bifurcation diagram and Lyapunov exponent of normalized microbubble radius are demonstrated by taking the shear modulus of the surrounding medium as the control parameter (figure 3 ) whereas the acoustic pressure amplitude is $1.5 \mathrm{MPa}$ and the ultrasound frequency is $1.5 \mathrm{MHz}$. Moreover, bifurcation diagrams and Lyapunov exponent of normalized microbubble radius versus acoustic pressure (figures 4(a)-(b)) and applied frequency (figures 5(a)-(b)) are plotted within the soft tissue and proportionately stiff tissue with $G_{L}=0.5$ and $1 \mathrm{MPa}$, respectively. 


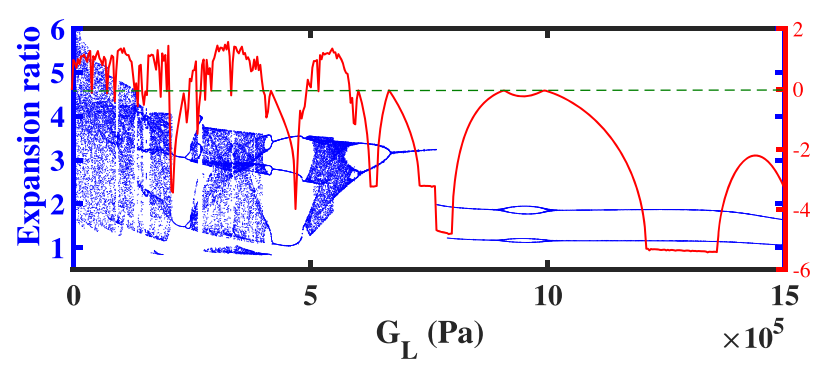

Figure 3. Bifurcation diagrams (Expansion ratio-blue dot points) and Lyapunov exponent ( $\lambda$-red solid line) of normalized microbubble radius versus shear modulus of surrounding medium whit $\mu_{s}=0.45 \mathrm{~Pa} \mathrm{~s}$, and $G_{S}=11.7 \mathrm{MPa}$ when the driving frequency and pressure are, respectively, $1.5 \mathrm{MHz}$ and $1.5 \mathrm{MPa}$. Here we have different regions: blood $G_{L}=0 \mathrm{MPa}$, soft tissue $G_{L} \leqslant 0.5 \mathrm{MPa}$, and stiff tissue $G_{L} \geqslant 1.5 \mathrm{MPa}$.

It is observed that the microbubble behavior is chaotic for low values of shear modulus (figure 3 ) and as the shear modulus of the outer medium is increasing the expansion ratio of the microbubble and chaotic pulsations are reducing. The microbubble finally goes to the stability by increasing the magnitude of shear modulus of the surrounding medium up to $765 \mathrm{kPa}$ which can be confirmed in [40].

In the same conditions, the microbubble behavior is probed versus acoustic pressure for two different values of the shear modulus of the surrounding medium, i.e., 0.5 and $1 \mathrm{MPa}$ (see figures 4(a)-(b)). Comparing these figures in figure 1(c) reveals that the oscillations abate by increasing the magnitude of the shear modulus of the medium; indeed the system has more stability when the external medium is more rigid. The microbubble demonstrates various dynamical behaviors in 3 values of $G_{L}$. When the microbubble is surrounded by blood with $G_{L}=0$ (see figure 1(c)), it undergoes more chaotic oscillations in lower pressure amplitudes, e.g., the first chaotic window is indicated in $677 \mathrm{kPa}$, this incident takes place in 1.27 MPa beside soft tissue (Figure 4(a)) and the system is completely stable for the case of comparatively hard tissue with $G_{L}=1 \mathrm{MPa}$ (figure 4(b)).

The effects of frequency variations on microbubble behavior for the values of $G_{L}=0.5,1 \mathrm{MPa}$ are plotted when the acoustic pressure is $1.7 \mathrm{MPa}$ (figures 5(a)-(b)). Comparing these results with figure 2(e) which shows the effect of frequency variations in $G_{L}=0$ manifests this fact that by increasing the magnitude of shear modulus of the surrounding medium, the chaotic oscillations decrease significantly and as it is seen in (figure 5(b)) the chaotic behavior disappears for $G_{L}=1 \mathrm{MPa}$. It is also evident that the expansion ratio of the microbubble is smaller for higher magnitudes of $G_{L}$, in fact, the nonlinearity intensifies for smaller values of $G_{L}$.

During our investigation, to explore the effect of shell viscosity alterations on microbubble dynamics, bifurcation diagrams and Lyapunov exponent of normalized microbubble radius are plotted versus shell viscosity as the control parameter. Its value varies in the range 0.01 to $5 \mathrm{~Pa}$ s for 3 values of $G_{L}(0,0.5,1 \mathrm{MPa})$ in frequency $=1 \mathrm{MHz}$ and acoustic pressure $=1.5 \mathrm{MPa}$.
Results represent that the expansion ratio of the microbubble is much higher for low values of shell viscosity in $G_{L}=0$ (figure 6(a)) and also it is evident that by increasing the value of shell viscosity the nonlinearity and the maximum microbubble expansion decrease which is seen in $[28,32,58]$. Figure 6(b) demonstrates the normalized oscillations of the microbubble versus time in frequency $=1 \mathrm{MHz}$ and acoustic pressure $=1.5 \mathrm{MPa}$ when the microbubble is surrounded by blood with $G_{L}=0$. This figure represents the chaotic oscillations of the microbubble for a definite value of the shell viscosity, i.e., $0.45 \mathrm{~Pa} \mathrm{~s}$ and as it is seen the maximum expansion ratio in this value is the same as figure 6(a).

The microbubble exhibits fully chaotic behavior for small values of shell viscosity which is pursued by period doubling and the system reaches to period one stability in $0.66 \mathrm{~Pa}$.s for $G_{L}=0.5 \mathrm{MPa}$. The UCA dynamics is completely stable in $G_{L}=1 \mathrm{MPa}$ when the UCA is surrounded by relatively stiff tissue (results was not shown here).

Next, by employing the values of $1.5 \mathrm{MHz}$ and $1 \mathrm{MPa}$ for driving frequency and pressure, respectively, but this time considering the shear modulus of the shell as the control parameter while varying between 0 to $200 \mathrm{MPa}$, the bifurcation diagrams are presented for 3 values of $G_{L}(0,0.5,1 \mathrm{MPa})$.

By figures 7 (a)-(c), the microbubble response is entirely disparate in $G_{L}=0$ (figure $7(\mathrm{a})$ ) with regards to $G_{L}=0.5 \mathrm{MPa}$ (Figure 7(b)) and $G_{L}=1 \mathrm{MPa}$ (figure 7(c)). Its dynamics is stable for the values of $G_{L}=0.5$ and $1 \mathrm{MPa}$ (figures 7(b)-(c)) while it exhibits chaotic oscillations and high expansion ratio in $G_{L}=0$ and the chaotic region becomes narrower in $G_{S}=171 \mathrm{MPa}$ (figure 7(a)).

One of the most important parameters that influence the microbubble behavior is the shell thickness of the microbubble which is utilized as the control parameter and varying in the range of 0 to $150 \mathrm{~nm}[28,31,32]$ with the values of frequency and pressure of $1 \mathrm{MHz}$ and $1.5 \mathrm{MPa}$, respectively. The bifurcation diagrams are sketched for three values of $G_{L}$ $(0,0.5,1 \mathrm{MPa})$.

Figure 8(a) exposes that the UCA endures chaotic pulsations in a small magnitude of the shell thickness and increasing the shell thickness decreases the expansion ratio of the microbubble diameter and the system becomes stable when the shell thickness of the agent is $124 \mathrm{~nm}$. These results confirm the previous works in a very wide range of shell thickness variations [28, 31, 32]. Figure 8(b) presents the corresponding time series of the normalized oscillations of the microbubble in frequency $=1 \mathrm{MHz}$ and acoustic pressure $=1.5 \mathrm{MPa}$ while the shell thickness of the agent is $50 \mathrm{~nm}$ and the microbubble is surrounded by blood with $G_{L}=0$. It is evident that the amplitude of pulsations is the same value as figure $8(\mathrm{a})$.

For $0.5 \mathrm{MPa}$ of $G_{L}$ the microbubble dynamics is chaotic for a small interval of shell thickness up to $18 \mathrm{~nm}$ and goes to the stable manner which lasts as a predominant situation to the end of the interval. For $1 \mathrm{MPa}$ of $G_{L}$ the microbubble remains stable in the whole range of shell thickness and any chaotic behaviors is not viewed (results was not shown here).

The stable domains of the polymer-shelled agent are summarized in table 3 for some consequential parameters. 

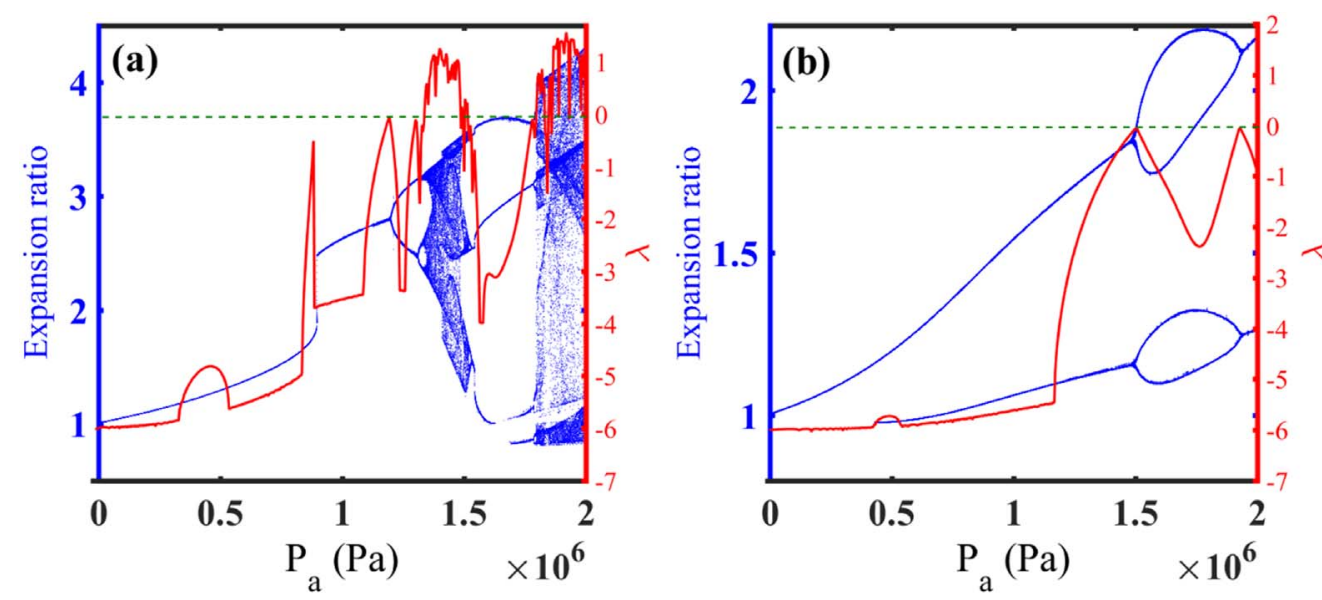

Figure 4. Bifurcation diagrams (Expansion ratio-blue dot points) and Lyapunov exponent ( $\lambda$-red solid line) of normalized microbubble radius versus driving pressure whit $\mu_{s}=0.45 \mathrm{~Pa} \mathrm{~s}$, and $G_{S}=11.7 \mathrm{MPa}$ when the applied frequency is $1.5 \mathrm{MHz}$ for the surrounding medium with $G_{L}$ (a) $0.5 \mathrm{MPa}$, (b) $1 \mathrm{MPa}$.
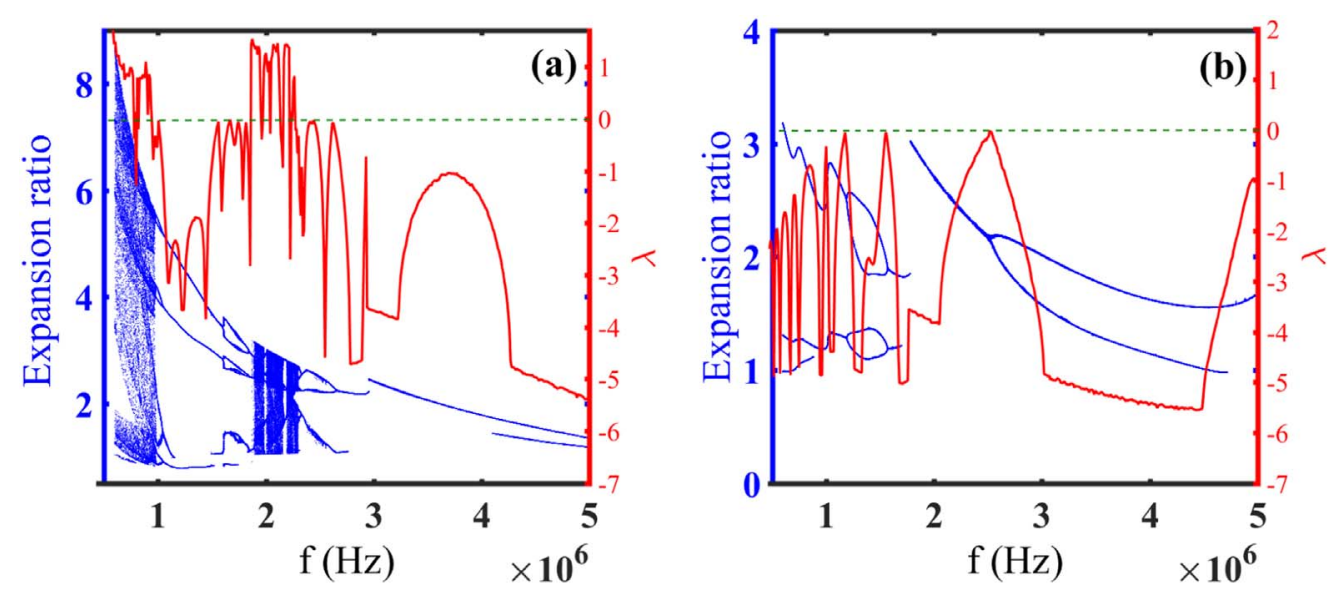

Figure 5. Bifurcation diagrams (Expansion ratio-blue dot points) and Lyapunov exponent ( $\lambda$-red solid line) of normalized microbubble radius versus driving frequency whit $\mu_{s}=0.45 \mathrm{~Pa} \mathrm{~s}$, and $G_{S}=11.7 \mathrm{MPa}$ when the acoustic pressure is $1.7 \mathrm{MPa}$ for the surrounding medium with $G_{L}$ (a) $0.5 \mathrm{MPa}$, (b) $1 \mathrm{MPa}$.

These results reveal that the stiffness, of the surrounding medium influences the UCA behavior impressively and also demonstrates the chaotic oscillations of UCA under the action of an ultrasound field which can be used to distinguish stable and unstable regions of microbubble pulsations and the expansion ratio of the UCA.

\section{Conclusions}

This article explained the dynamics driven a polymer-shelled gas microbubble submerged in soft tissue by using the techniques of chaos physics and the ranges in which microbubble has stable behavior has been shown and also been tabulated to show stability limits of the microbubble, which is extremely important in applications. Actually, in order to understand the behavior of an UCA in sonified by high intensity ultrasound in therapeutic medicine in vivo, it is necessary to model the UCA as a shelled gas bubble surrounded by soft tissue. In this paper, we used this model because Qin-Ferrara [40] have presented this model to explain the radial oscillations of
UCAs in vivo while by considering the effects of the surrounding tissue, liquid compressibility, and the shell. Results of the radial motion of a polymer-shelled agent display that Qin-Ferrara model which reported in this paper is capable of capturing the essential features of the drug and gene delivery applications. The comprehension of UCA behavior is indispensable to improve its diagnostic and therapeutic implementations in which the nonlinearities cannot be prevented. Nonlinear oscillations of encapsulated microbubble immersed in blood or tissue are scrutinized. The complex dynamics of the microbubble is examined in high acoustic pressure amplitudes with the great magnitude of pulsations which is prevalently utilized in drug and gene delivery applications. The effects of several significant parameters on the behavior of the agent are shown for a wide range of variations which has not been inspected previously. These results provide an exact and comprehensive insight into the system dynamics versus a spacious domain of control parameters i.e. pressure and frequency thresholds for stability in blood are summarized in table 3. By focusing on the mechanisms governing the transition from the chaotic oscillations to the stable region, 

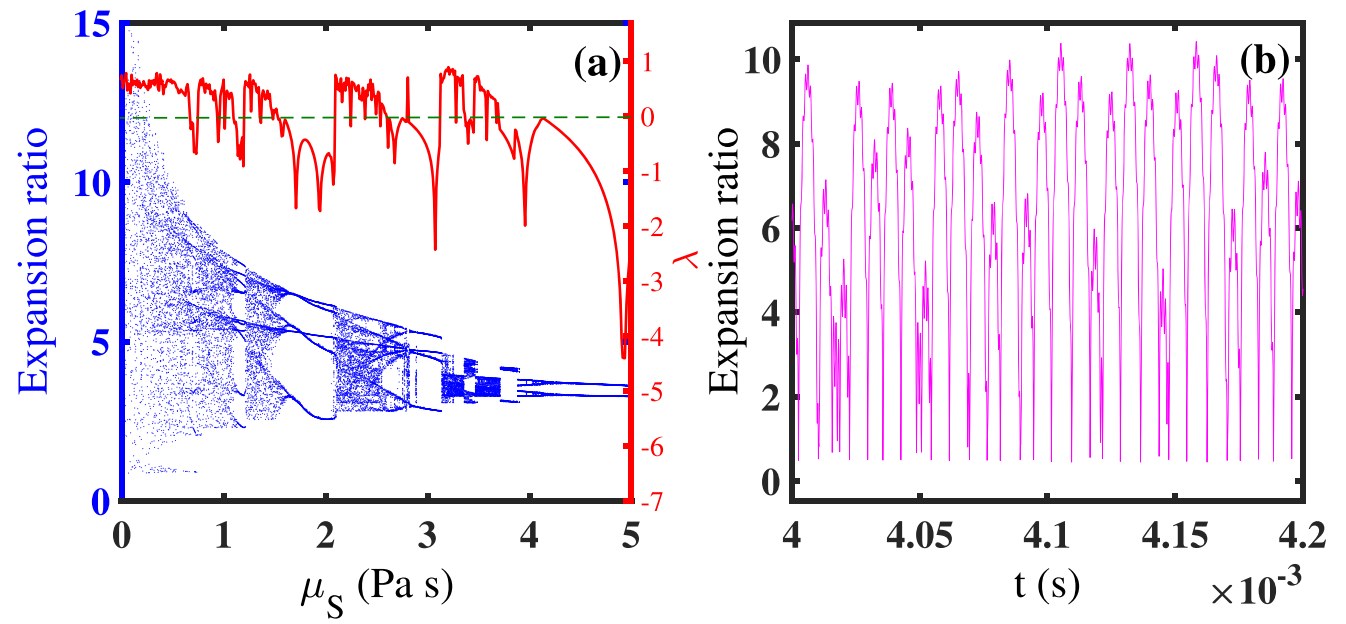

Figure 6. (a) Bifurcation diagrams (Expansion ratio-blue dot points) and Lyapunov exponent ( $\lambda$-red solid line) of normalized microbubble radius versus shell viscosity when the driving frequency and pressure are, respectively, $1 \mathrm{MHz}$ and $1.5 \mathrm{MPa}$ for $G_{L}=0$, and $G_{S}=11.7 \mathrm{MPa}$, (b) The corresponding time series of normalized oscillations with the shell viscosity $\mu_{s}=0.45 \mathrm{~Pa}$.
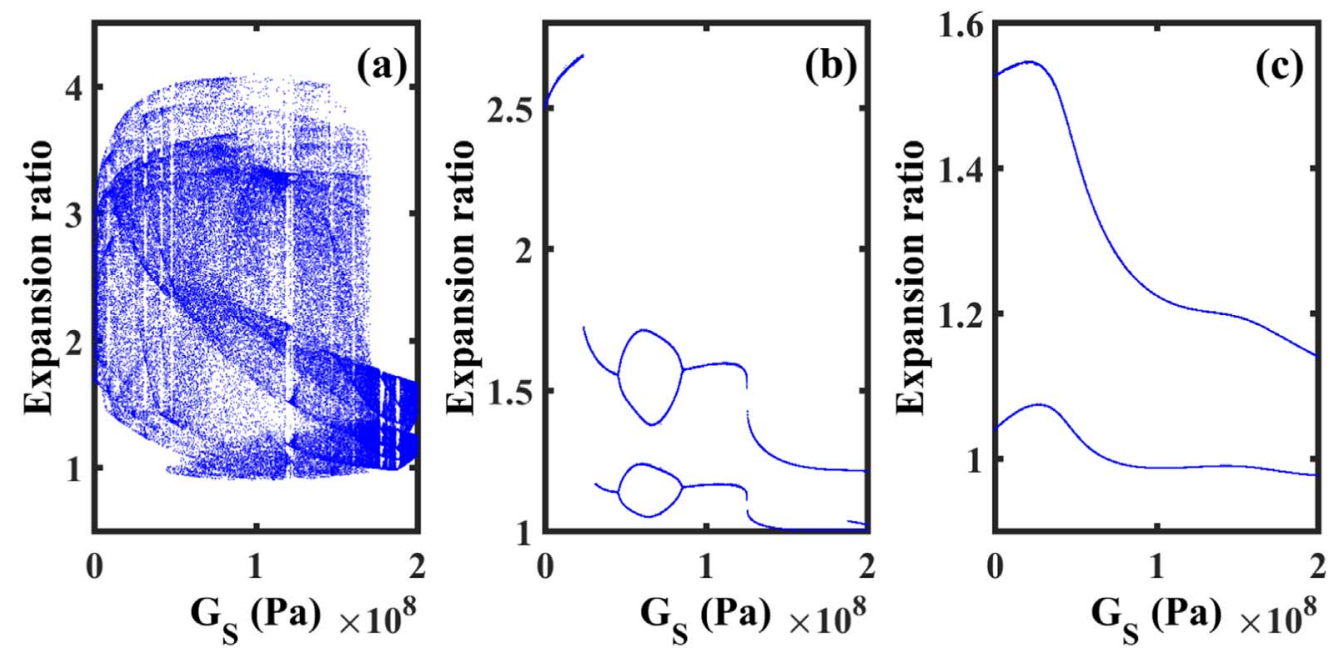

Figure 7. Bifurcation diagrams of normalized microbubble radius versus shear modulus of shell when the driving frequency and pressure are, respectively, $1.5 \mathrm{MHz}$ and $1 \mathrm{MPa}$ for $\mu_{s}=0.45 \mathrm{~Pa} \mathrm{~s}, G_{S}=11.7 \mathrm{MPa}$, and $G_{L}$ is (a) $0 \mathrm{MPa}$, (b) $0.5 \mathrm{MPa}$, (c) $1 \mathrm{MPa}$.
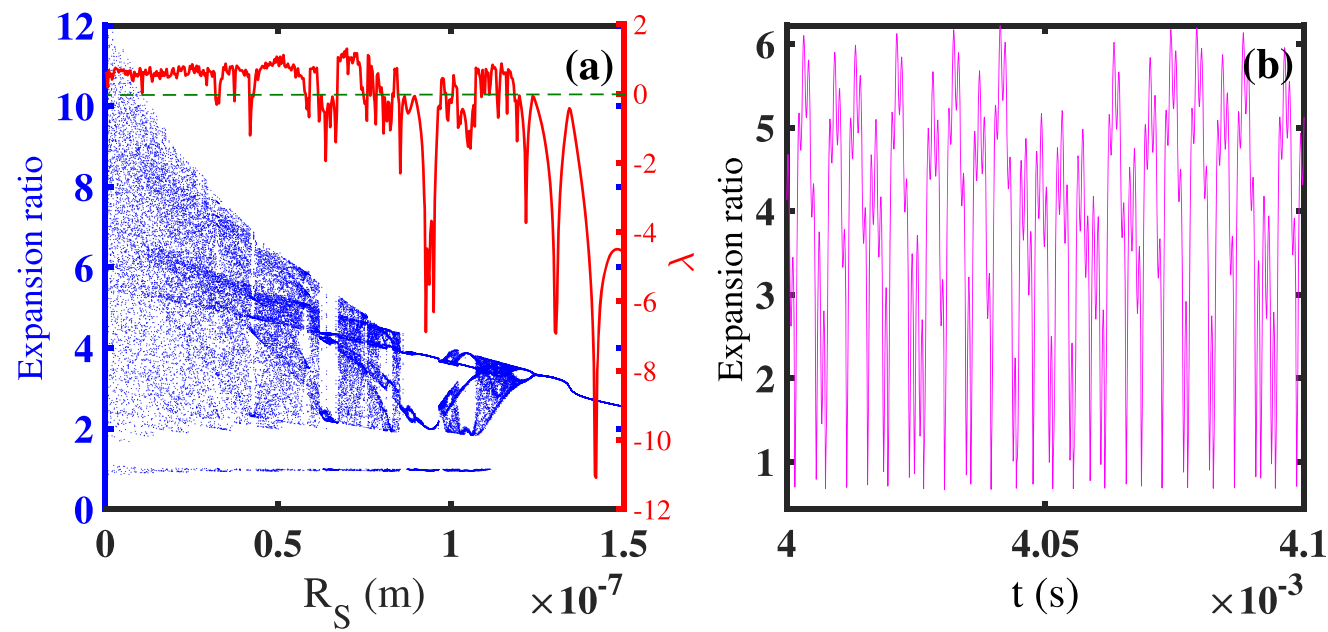

Figure 8. (a) Bifurcation diagrams (Expansion ratio-blue dot points) and Lyapunov exponent ( $\lambda$-red solid line) of normalized microbubble radius versus shell thickness when the driving frequency and pressure are, respectively, $1 \mathrm{MHz}$ and $1.5 \mathrm{MPa}$ for $\mu_{s}=0.45 \mathrm{~Pa} \mathrm{~s}$, $G_{S}=11.7 \mathrm{MPa}$, and $G_{L}=0$, (b) The corresponding time series of normalized oscillations with the shell thickness. $\mathrm{R}_{S}=50 \mathrm{~nm}$. 
Table 3. Stable regions of polymer-shelled agent versus variations of various parameters in $G_{L}=0$ (blood), 0.5 (soft tissue) and 1.5 (stiff tissue) $\mathrm{MPa}$.

\begin{tabular}{lcccc}
\hline Parameter & $G_{L}=0$ & $G_{L}=0.5$ & $G_{L}=1.5$ & Unit \\
\hline Pressure & $<0.65$ & $<1.3$ & entirely stable & $\mathrm{MPa}$ \\
Frequency & $>2.65$ & $>2.45$ & entirely stable & $\mathrm{MHz}$ \\
Shell viscosity & $>2.82$ & $>0.53$ & entirely stable & $\mathrm{Pa} \mathrm{s}$ \\
Shell thickness & $>124$ & $>18$ & entirely stable & $\mathrm{nm}$ \\
Shear modulus of shell & $>171$ & entirely stable & entirely stable & $\mathrm{MPa}$ \\
\hline
\end{tabular}

this study opens a new horizon in studying the chaotic behavior of nonlinear dynamics of a shelled gas bubble submerged in soft tissue or blood.

\section{ORCID iDs}

M Yahyavi (iD https://orcid.org/0000-0003-0062-203X

\section{References}

[1] Decuzzi P, Godin B, Tanaka T, Lee S-Y, Chiappini C, Liu X and Ferrari M 2010 Size and shape effects in the biodistribution of intravascularly injected particles $J$ of Controlled Release 141 320-7

[2] Sirsi S R and Borden M A 2009 Microbubble compositions, properties and biomedical applications Bubble Sci. Eng. Technol. 1 3-17

[3] Borrelli M J, O'Brien W D Jr, Bernock L J, Williams H R, Hamilton E, Wu J, Oelze M L and Culp W C 2012 Production of uniformly sized serum albumin and dextrose microbubbles Ultrason. Sonochem. 19 198-208

[4] Mitterberger M, Pelzer A, Colleselli D, Bartsch G, Strasser H, Pallwein L, Aigner F, Gradl J and Frauscher F 2007 Contrast-enhanced ultrasound for diagnosis of prostate cancer and kidney lesions Eur. J. Radiol. 64 231-8

[5] Klibanov A L 2006 Microbubble contrast agents: targeted ultrasound imaging and ultrasound assisted drug-delivery applications Invest. Radiol. 41 354-62

[6] Shohet R V and Grayburn P A 2006 Potential bioeffects of ultrasonic destruction of microbubble contrast agents $J$ of the American College of Cardiology 47 1469-70

[7] Hynynen K 2008 Ultrasound for drug and gene delivery to the brain Adv. Drug Delivery Rev. 60 1209-17

[8] Sirsi S R, Hernandez S L, Zielinski L, Blomback H, Koubaa A, Synder M, Homma S, Kandel J J, Yamashiro D J and Borden M A 2011 Polyplex-microbubble hybrids for ultrasound-guided plasmid DNA delivery to solid tumors J. Controlled Release 157 224-34

[9] Porter T R and Xie F 2001 Ultrasound, microbubbles, and thrombolysis Prog. Cardiovasc. Dis. 44 101-10

[10] Hynynen K, McDannold N, Vykhodtseva N, Jolesz F and Noninvasive $2001 \mathrm{MR}$ imaging-guided focal opening of the blood-brain barrier in rabbits Radiology 220 640-6

[11] Yang F Y, Fu W M, Yang R S, Liou H C, Kang K H and Lin W L 2007 Quantitative evaluation of focused ultrasound with a contrast agent on blood-brain barrier disruption Ultrasound Med. Biol. 33 1421-7

[12] McDannold N J, Vykhodtseva N I and Hynynen K 2006 Microbubble contrast agent with focused ultrasound to create brain lesions at low power levels: MR imaging and histologic study in rabbits Radiology 241 95-106
[13] Lentacker I, De Smedt S C and Sanders N N 2009 Drug loaded microbubble design for ultrasound triggered delivery Soft Matter 5 2161-70

[14] Bull J L 2007 The application of microbubbles for targeted drug delivery Expert. Opin. Drug Deliv. 4 475-93

[15] Sheffield P, Trehan A, Boyd B and Wong O L 2008 Microbubbles as ultrasound contrast agents and in targeted drug delivery Crit. Rev. Biomed. Eng. 36 225-55

[16] Böhmer M R, Chlon C H T, Raju B I, Chin C T, Shevchenko T and Klibanov A L 2010 Focused ultrasound and microbubbles for enhanced extravasation J. Controlled Release 148 18-24

[17] Unger E C, Porter T, Culp W, Labell R, Matsunaga T and Zutshi R 2004 Therapeutic applications of lipid-coated microbubbles Adv. Drug Deliv. Rev. 56 1291-314

[18] Ferrara K, Pollard R and Borden M 2007 Ultrasound microbubble contrast agents: fundamentals and application to gene and drug delivery Аnnu. Rev. Biomed. Eng. 9 415-47

[19] Wu J, Pepe J and Dewitt W 2003 Nonlinear behaviors of contrast agents relevant to diagnostic and therapeutic applications Ultrasound Med. Biol. 29 555-62

[20] Chomas J E, Dayton P, May D and Ferrara K 2001 Threshold of fragmentation for ultrasonic contrast agents J. Biomed. Opt. 6 141-50

[21] Morgan K E, Allen J S, Dayton P A, Chomas J E, Klibanov A L and Ferrara K W 2000 Experimental and theoretical evaluation of microbubble behavior: effect of transmitted phase and bubble size IEEE Transact. Ultrasonics Ferroelect. Freq. Control. 47 1494-509

[22] Hoff L, Sontum P C and Hovem J M 2000 Oscillations of polymeric microbubbles: effect of the encapsulating shell J. Acoust. Soc. Am. 107 2272-80

[23] Lauterborn W and Koch A 1987 Holographic observation of period-doubled and chaotic bubble oscillations in acoustic cavitation Phys Rev A 35 1974-6

[24] Dzaharudin F, Suslov S A, Manasseh R and Ooi A 2013 Effects of coupling, bubble size, and spatial arrangement on chaotic dynamics of microbubble cluster in ultrasonic fields $J$ Acoust Soc Am. 134 3425-34

[25] Chong K J Y, Quek C Y, Dzaharudin F, Ooi A and Manasseh R 2010 The effects of coupling and bubble size on the dynamical-systems behaviour of a small cluster of microbubbles J Sound Vib 329 687-99

[26] De Jong N 1993 Acoustic properties of ultrasound contrast agents Ph.D. Thesis http://hdl.handle.net/1765/39138 Erasmus University, Rotterdam, The Netherlands

[27] De Jong N and Hoff L 1993 Ultrasound scattering of Albunex microspheres Ultrasonics 31 175-81

[28] Church C C 1995 The effects of an elastic solid surface layer on the radial pulsations of gas bubbles J. Acoust. Soc. Am. 97 1510-21

[29] Morgan K E 2001 Experimental and theoretical evaluation of ultrasonic contrast agent behavior Ph.D. Thesis University of Virginia, Ann Arbor, USA 
[30] Zheng H, Dayton P A, Caskey C, Zhao S, Qin S and Ferrara K W 2007 Ultrasound driven microbubble oscillation and translation within small phantom vessels Ultrasound Med. Biol. 33 1978-87

[31] Allen J S, May D J and Ferrara K W 2002 Dynamics of therapeutic ultrasound contrast agents Ultrasound Med. Biol. 28 805-16

[32] Khismatullin D B and Nadim A 2002 Radial oscillations of encapsulated microbubbles in viscoelastic liquids Phys. Fluids 14 3534-57

[33] Chatterjee D and Sarkar K 2003 A Newtonian rheological model for the interface of microbubble contrast agents Ultrasound Med. Biol. 29 1749-57

[34] Sarkar K, Shi W T, Chatterjee D and Forsberg F 2005 Characterization of ultrasound contrast microbubbles using in vitro experiments and viscous and viscoelastic interface models for encapsulation J. Acoust. Soc. Am. 118 539-50

[35] Stride E and Saffari N 2004 Theoretical and experimental investigation of the behavior of ultrasound contrast agent particles in whole blood Ultrasound Med. Biol. 30 1495-509

[36] Tamadapu G, Grishenkov D and Eriksson A 2016 Modeling and parametric investigation of thick encapsulated microbubble's nonspherical oscillations J. Acoust. Soc. Am. 1403884

[37] Tamadapu G, Nordmark A and Eriksson A 2015 Resonances of a submerged fluid-filled spherically isotropic microsphere with partial-slip interface condition J. Appl. Phys. 118 044903

[38] Marmottant P, van der Meer S, Emmer M, Versluis M, de Jong N, Hilgenfeldt S and Lohse D 2005 A model for large amplitude oscillations of coated bubbles accounting for buckling and rupture J. Acoust. Soc. Am. 118 3499-505

[39] Doinikov A and Dayton P A 2006 Spatio-temporal dynamics of an encapsulated gas bubble in an ultrasound field $J$ Acoust Soc Am. 120 661-9

[40] Qin S and Ferrara K W 2010 A model for the dynamics of ultrasound contrast agents in vivo J Acoust Soc Am. 128 1511-21

[41] Frinking P, Segers T, Luan Y and Tranquart F 2020 Three Decades of Ultrasound Contrast Agents: A Review of the Past, Present and Future Improvements Ultrasound in Medicine \& Biology 46 892-908

[42] Helfield B 2019 A review of phospholipid encapsulated ultrasound contrast agent microbubble physics Ultrasound Med. Biol. 45 282-300

[43] Lauterborn W and Parlitz U 1988 Methods of chaos physics and their application to acoustics $J$ Acoust Soc Am $\mathbf{8 4}$ 1975-93
[44] Parlitz U, Englisch V, Scheffczyk C and Lauterborn W 1990 Bifurcation structure of bubble oscillators J Acoust Soc Am. 88 1061-77

[45] Parlitz U, Scheffczyk C, Akhatov I and Lauterborn W 1995 Structure formation in cavitation bubble fields Chaos, Solitons Fractals 5 1881-91

[46] Lauterborn W and Suchal E 1984 Bifurcation superstructure in a model of acoustic turbulence Phys. Rev. Lett. 53 2304-7

[47] Behnia S, Zahir H, Yahyavi M, Barzegar A and Mobadersani F 2013 Observations on the dynamics of bubble cluster in an ultrasonic field Nonlinear Dyn. 72 561-74

[48] Behnia S, Yahyavi M and Mobadersani F 2014 Intelligent controlling microbubble radial oscillations by using SlaveMaster Feedback control Appl. Math. Comput. 245 404-15

[49] Behnia S, Mobadersani F, Yahyavi M and Rezavand A 2013 Chaotic behavior of gas bubble in non-Newtonian fluid: a numerical study Nonlinear Dyn. 74 559-70

[50] Behnia S, Mobadersani F, Yahyavi M, Rezavand A, Hoesinpour N and Ezzat A 2015 Effect of magnetic field on the radial pulsations of a gas bubble in a non-Newtonian fluid Chaos, Solitons Fractals 78 194-204

[51] Brenner M P, Hilgenfeldt S and Lohse D 2002 Single-bubble sonoluminescence Rev. Mod. Phys. 74 425-84

[52] Wolf A, Swift J B, Swinney H L and Vastano A 1985 Determining the Lyapunov exponents from a time series Physica D 16 285-317

[53] Sarkara K, Shi W T and Chatterjee D 2005 Flemming Forsberg, Characterization of ultrasound contrast microbubbles using in vitro experiments and viscous and viscoelastic interface models for encapsulation $J$ Acoust Soc Am. 118 539-50

[54] Sboros V 2008 Response of contrast agents to ultrasound $A d v$. Drug Delivery Rev. 60 1117-36

[55] King D A and Orien W D Jr. 2011 Comparison between maximum radial expansion of ultrasound contrast agents and experimental postexcitation signal results J. Acoust. Soc. Am. 129 114-21

[56] Zheng H, Dayton P A, Casky C, Zhao S, Qin S and Ferrara K W 2007 Ultrasound-driven microbubble oscillation and translation within small phantom vessels Ultrasound Med. Biol. 33 1978-87

[57] Kooiman K, Vos H J, Versluis M and de Jong N 2014 Acoustic behavior of microbubbles and implications for drug delivery Adv. Drug Delivery Rev. 72 28-48

[58] Doinikov A A, Haac J F and Dayton P A 2009 Modeling of nonlinear viscous stress in encapsulating shells of lipidcoated contrast agent microbubbles Ultrasonics 269-75 